\title{
Yttrium-90 Radioembolization for Hepatocellular Carcinoma Prior to Liver Transplantation: Reply
}

\author{
Giuseppe Maria Ettorre $^{1} \cdot$ Giovanni Battista Levi Sandri ${ }^{1} \cdot$ Giovanni Vennarecci $^{1}$
}

Published online: 5 May 2017

(C) Société Internationale de Chirurgie 2017

\section{Dear Editor,}

We read with attention the Letter to the Editor written by Goh BK. about our manuscript entitled "yttrium- 90 radioembolization for hepatocellular carcinoma prior to liver transplantation" [1]. We thanks prof. Goh for giving us the opportunity to clarify some important aspect of our study. As specified the aim of our study was to retrospectively analyze patients transplanted for hepatocellular carcinoma (HCC) previously treated with yttrium-90 radioembolization (Y90-RE). From January 2002 to December 2015, 22 patients were transplanted after Y90RE. Patients treated with Y90-RE were strictly followed up and included into the waiting list after a mean period of $8-12$ months. The radiological results of the 22 transplanted patients was positive in 18 cases $(81.8 \%$ ) (patients were radiologically considered Milan in after Y90-RE); of them 15 patients $(78.9 \%)$ had a successful radiological downstaging and three patients $(100 \%)$ had a successful bridge to liver transplant (LT). On the other hand, we calculate the pathological response on the 22 livers and we found that 11 patients $(57.8 \%)$ had an effective downstaging and 2 patients $(66.6 \%)$ an effective bridge to LT. This results mean that we moved from a radiological expectation response from 100 to $66.6 \%$ for the bridge treatment and from 78.9 to $57.8 \%$ for the downstaging therapy.

Giuseppe Maria Ettorre

gmettorre@scamilloforlanini.rm.it

$\triangle$ Giovanni Battista Levi Sandri

gblevisandri@gmail.com

1 Division of General Surgery and Liver Transplantation, San Camillo Hospital Rome, circ.ne Gianicolense 87,

00151 Rome, Italy
As Goh correctly underline at the publication date, we had 7 patients under evaluation and 2 were in the waiting list which means that they had a positive response to therapy. To date we transplanted others 3 patients after a downstaging Y90-RE and 4 are in waiting list. So looking at the previous rates, we can tell that from 41 patients treated in a downstaging to transplant intent, $78 \%(32 / 41)$ had a downstaging with Y90-RE.

Concerning the bridge therapy, recently a prospective, randomized phase 2 study described Y90-RE to have significantly longer time to progression compared to transarterial chemoembolization [2]. In case of HCC patients in a liver transplantation setting a bridge therapy should be performed with Y90-RE when necessary [3].

We believe that with this clarification letter, casual reader may have no doubt that Y90-RE is an important an interesting therapy for patients in the field of transplantation.

\section{References}

1. Ettorre GM, Sandri GBL, Laurenzi A et al (2017) Yttrium-90 Radioembolization for Hepatocellular Carcinoma Prior to Liver Transplantation. World J Surg 41:241-249. doi:10.1007/s00268016-3682-Z

2. Salem R, Gordon AC, Mouli S et al (2016) Y90 Radioembolization significantly prolongs time to progression compared with chemoembolization in patients with hepatocellular carcinoma. Gastroenterology 151(6):1155-1163. doi:10.1053/j.gastro.2016. 08.029

3. Levi Sandri GB, Ettorre GM (2016) An ace in the hole for hepatocellular carcinoma: yttrium-90 radioembolization. Transl Cancer Res 5(Suppl 6):S1055-S1056. doi:10.21037/tcr.2016.11.08 